\title{
Developing Semantically Interoperable PH Emergency Preparedness Data Exchange
}

\author{
Nikolay Lipskiy*, James Tyson and Jaqueline Burkholder \\ CSELS/DHIS, CDC, Atlanta, GA, USA
}

\section{Objective}

The purpose of this project is to demonstrate progress in developing functional data models and semantic definitions (content standards) for data elements and value sets comprising information categories supporting PH Emergency Preparedness and Response (EPR). The objective is to explain the concepts and methods used to define core $\mathrm{PH}$ Emergency Management and Preparedness and Response functions, Information Exchange Requirements (IERs), data elements, and value sets to create a PH Emergency Operations Center (EOC) Minimum Data Set Specification. The primary focus of this presentation is to describe the value of semantic data interoperability and provide operational examples of the value and return-on-investment gained through building semantically interoperable data exchange through content standardization.

\section{Introduction}

Effective prevention, detection, and rapid response to $\mathrm{PH}$ emergencies rely on sufficient and timely delivered information. PH EOC data flows are based on critical information requirements, addressing needs of EOC staff for timely delivered analytical products that provide situational awareness, event-specific data, event investigation tools, resource management etc ${ }^{1}$. The ability of PH EOC systems to automatically and accurately interpret meaning of the exchanged data depends on a level of semantic data interoperability and utilization of a common information exchange reference model (CIERF) that conforms to established data standards. PH EOC data interoperability requires mutual development and close collaboration with partners to develop a PH EPR CIERF, common terminology and standardized vocabulary.

\section{Methods}

The CDC's Situational Awareness Branch (SAB) facilitates national activities on development $\mathrm{PH}$ EOC informatics through participation in the WHO EOC Network (EOC-NET) ${ }^{2}$, and collaboration with national organizations and CDC partners on content standardization. The following sources were used for this analysis: 1 ) 26 content standards developed by national and international standard development organization, 2) WHO's Framework for a Public Health Emergency Operations Centre ${ }^{2}$, and 3) PH EOC data requirements ${ }^{3}$ that were published by $\mathrm{CDC}$ 's $\mathrm{SAB}$. These data requirements were included into the CDC Vocabulary and Access Distribution System (VADS $)^{4}$, which serves as the primary vocabulary content browser for PH EPR informatics.

\section{Results}

In analyzing the PH EPR content standards, the CDC's SAB arrived at the following results. The CDC EOC's process of development and implementation content standards is based on the PH EOC critical information requirements. These requirement became business rules for the PH EPR CIERF.

The current, version 2, of the PH EPR CIERF consists of 12 information modules including PH EOC minimum data set (MDS), patient clinical observations, emergency medical systems (EMS), data elements for emergency departments (DEEDS), WHO MDS for Health Workforce Registry, Resource Utilization Message Component (vocabulary for hospital resources), vocabulary for the national trauma standard. These PH EPR CIERF modules are interoperable and built on existing data standards. These modules were codified by VADS and ready for utilization by international and national PH EOC partners. At the stage of this analysis the PH EPR CIERF codification schema was prepared for adding it into the Logical Observation Identifiers Names and Codes (LOINC) content standard.

The current PH EOC MDS version was released in September 2017. The common terminology and vocabulary that were included into this version are conformant with existing national and international content standards and specifications. Comparatively to the previous version 1, the current PH EOC MDS contains more than $60 \%$ new and updated terminology and value sets.

Added to the PH EOC MDS version 2 new features are the Situational Analysis concept model, that also incorporates a nomenclature and structure for the Public Health EOC Situational Report (SITREP). Also, the Managing and Commanding conceptual model was updated by adding concepts and vocabulary for the agency internal communication, including standardized knowledge repository for managing standard operating procedures (SOP) and reports for leadership.

The CDC's SAB directly supports the CDC Surveillance Data Platform (SDP) and national organizations on development of electronic forms and form builders. These efforts will provide additional capabilities for collecting and electronically sharing standardized SA information utilizing web-enabled Services and mobile capabilities.

\section{Conclusions}

CDC's EOC and Division of Emergency Operations Staff is improving the application of emergency management and $\mathrm{PH}$ practice in preparing and responding to emergencies through partnerships and coordinated work with Standard Development Organizations (SDOs) to add critical EPR vocabularies to national and international standards. This work supports National Emergency Management Organizations and is a reference source for the WHO EOC-NET guiding documents supporting international efforts to strengthen Global Health Security.

\section{Keywords}

$\mathrm{PH}$ emergency preparedness; semantic interoperability; $\mathrm{PH}$ emergency information center informatics

\section{References}

1. Lipskiy N., Tyson J. Advancing PH Emergency Preparedness Informatics to Support Emergency Responses. Online J Public Health Inform. 2017; 9(1): e059. At: http://ojphi.org/ojs/index.php/ojphi/ article/download/7637/6158

2. WHO. Framework for a Public Health Emergency Operations Centre. Interim document. November, 2015. At: http://www.who.int/ihr/ publications/9789241565134_eng/en/

3. CDC. Public Health Emergency Preparedness. At: https://phinvads.cdc. gov/vads/SearchVocab.action

4. CDC. Public Health Information Network. Vocabulary Access and Distribution System (PHIN VADS). At: https://phinvads.cdc.gov/ vads/SearchVocab.action

\section{*Nikolay Lipskiy}

E-mail: dgz1@cdc.gov

ISDS Annual Conference Proceedings 2018. This is an Open Access article distributed under the terms of the Creative Commons AttributionNoncommercial 3.0 Unported License (http://creativecommons.org/licenses/by-nc/3.0/), permitting all non-commercial use, distribution, and reproduction in any medium, provided the original work is properly cited. 